\title{
Thrombotic Microangiopathy and Kidney Injury After Hematopoietic Stem Cell Transplantation
}

\author{
Fazilet Yilmaz, Vivek Kasinath, Merve Postalcioglu, \\ Vincent T. Ho, and Reza Abdi
}

\section{INTRODUCTION}

Transplant-associated thrombotic microangiopathy (TA-TMA) is a systemic condition in which a series of pathological injuries to the endothelial cells result in multiorgan failure. ${ }^{1}$ The reported incidence of TMA following hematopoietic stem cell transplantation (post-HSCT TMA) ranges between $0.5 \%$ and $63.6 \% .{ }^{2}$ This wide range reflects the wide variety of diagnostic approaches taken to define this condition. Postmortem biopsy, for instance, identifies TMA lesions more commonly than the clinical definitions of TMA, suggesting that this condition is underdiagnosed. ${ }^{3}$

A multitude of causes, including immunosuppressive regimens, infectious agents, and radiation, contribute to the pathogenesis of TA-TMA. Kidney failure is a typical complication of TA-TMA. However, accumulating evidence highlights the common involvement of other organs, including the lungs, gastrointestinal (GI) tract, heart, and central nervous system. ${ }^{1}$

TA-TMA is seen after both solid organ transplantation and HSCT, but post-HSCT TMA remains the most common form of TA-TMA. ${ }^{4}$ This review focuses on post-HSCTTMA, referred to henceforth as TA-TMA, and its link to kidney failure.

\section{DIAGNOSIS}

The diagnosis of TA-TMA is challenging, and the existing clinical criteria contain numerous pitfalls. As TMA typically occurs early following HSCT, renal biopsy is generally not possible due to lack of tolerability by the patient and the accompanying risk of bleeding. In addition, the possibility of patchy involvement of the kidney and intermittent nature of the TMA in some patients should be considered before proceeding to biopsy. No reliable biomarker exists to detect and confirm the diagnosis. In most cases, a diagnosis is made according to the presenting clinical signs and symptoms, notwithstanding the absence of a universal set of diagnostic 
criteria forTA-TMA. Notably, clinical suspicion allows for the early detection of TMA by the clinician. Elevated lactate dehydrogenase (LDH) levels, decreased platelet count, low haptoglobin, and the presence of schistocytes are included in different criteria for diagnosing TA-TMA (Table 13.1). However, these parameters are not sufficient to confirm the diagnosis. Haptoglobin, though expected to decrease in the setting of hemolysis, is an acute-phase reactant, so increases in production can balance the accompanying decline by consumption. Increased permeability may lead to the extravasation of schistocytes. ${ }^{1}$ Approximately $50 \%$ of the patients may have normal complement levels in the acute setting. ${ }^{5}$ Waiting for serum creatinine $(\mathrm{Cr})$ elevation or neurologic dysfunction could delay the diagnosis and will negatively affect therapy outcomes. Moreover, a delay in diagnosis of the condition could be deadly, so identifying early clinical findings can be crucial to the patient's outcome.

\section{PATHOGENESIS AND RISK FACTORS}

Injury to the endothelial cells that line the small blood vessels is the key underlying feature in the pathogenesis of TMA. HSCT patients are exposed a priori to procoagulant risk factors, such as chemotherapy, irradiation, prolonged immobilization, and infections. Following transplantation, calcineurin inhibitors (CNIs), mTOR inhibitors, total body irradiation (TBI), and infection propagate endothelial injury. Current studies demonstrate that damaged endothelial cells activate neutrophils by secreting interleukin-8 (IL-8), which leads to the formation of neutrophil extracellular traps (NETs). The formation of NETs activates the classical as well as alternative complement pathways, promotes inflammatory activation, and abets cell lysis. ${ }^{2,6}$ The inflammatory process encompasses activated T cells, B cells, monocytes, and macrophages, which secrete other cytokines and produce antibodies. The propagation of this inflammation activates intravascular platelets, which results in the formation of microthrombi, fragmentation of red blood cells, consumption of platelets, redistribution of blood flow, and increased permeability, ${ }^{1}$ leading to tissue ischemia. These changes can be confirmed by kidney biopsy, which reveals the presence of platelet-rich microthrombi, arteriolar and capillary thickening, subendothelial accumulation of proteins, and cell debris. ${ }^{6}$

The underlying complement-mediated injury to the endothelial cells in the kidney affects primarily the capillaries in the glomerulus and those that supply blood to the tubules. Another source of renal damage is hemolysis-related iron deposition and hemosiderosis, which can result in acute or chronic tubular injury. ${ }^{7}$ 


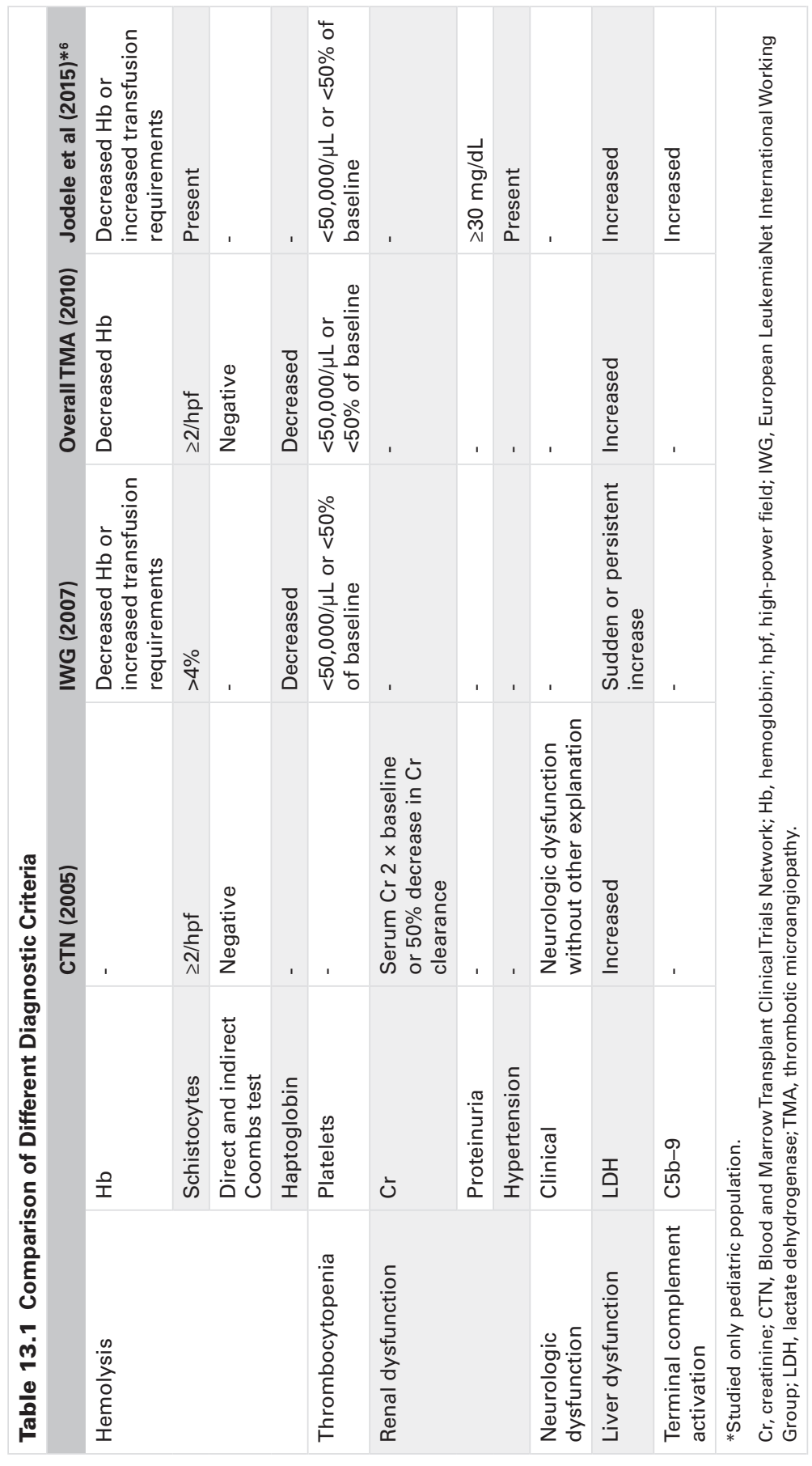




\section{SIGNS AND SYMPTOMS}

\section{Renal Manifestations}

The kidneys are the most common organs affected by TA-TMA, and the most significant complication in surviving patients is renal dysfunction. ${ }^{6}$ Renal involvement can manifest with decreased glomerular filtration rate (GFR), nephrotic range proteinuria, hypertension, end-stage renal disease (ESRD), or related death. Acute kidney injury (AKI) can develop secondary to ischemia during the disease course. Fifteen percent of the surviving definitive TMA patients need renal replacement therapy, and $30 \%$ of the patients have persistent renal dysfunction 6 months after transplantation. ${ }^{8}$ Uncontrolled hypertension may result in neurologic and cardiac complications.

HSCT patients are already at high risk for renal dysfunction due to their clinical condition and treatments. Volume depletion; systemic vasodilation due to sepsis; renal vasoconstriction; endothelial injury from graft-versus-host disease (GVHD), CNIs, orTBI; and tubular injury from conditioning chemotherapy or medications are risk factors for AKI among HSCT patients independent from TMA. Chronic renal dysfunction in TMA patients can be due directly to TMA, progression of pretransplant kidney disease, or CNI-related toxicity. Moreover, CNIs and steroids cause hypertension. If hypertension persists in spite of using two antihypertensive medications, TMA should be excluded. ${ }^{6}$

Monitoring renal function with serum $\mathrm{Cr}$ is important for early detection of TMA and exclusion of kidney injury due to other risk factors. For pediatric populations, other parameters for the estimation of GFR can be more reliable than serum $\mathrm{Cr}$.

\section{Manifestations of Other Organ Systems}

Central nervous system symptoms, such as headache, seizure, and confusion, can be observed in $30 \%$ of TA-TMA patients. ${ }^{9}$ Posterior reversible encephalopathy syndrome (PRES) can occur due to uncontrolled TMA-associated hypertension. ${ }^{6}$ It manifests with headache, visual changes, altered consciousness, and seizure. ${ }^{10}$ In neuroimaging, bleeding can be observed in the posterior cerebrum, brainstem, cerebellum, or basal ganglia. ${ }^{6}$

Several studies demonstrate pulmonary involvement inTA-TMA. Damage to pulmonary vessels increases pulmonary arterial pressure and results in right ventricular failure. Patients with hypoxia and pulmonary stress can undergo transthoracic echocardiography to test for pulmonary hypertension. ${ }^{6}$ Patients may also have $\mathrm{GI}$ symptoms like vomiting and diarrhea, and ischemic bowel changes in TA-TMA can cause abdominal pain and bleeding, which must be differentiated from sequelae of gut GVHD. ${ }^{6}$ A recent case report 
demonstrated an isolated intestinalTA-TMA patient who presented with $\mathrm{Gl}$ bleeding, confirmed by postmortem biopsy. ${ }^{3}$

In addition, unexplained pericardial, pleural, or peritoneal fluid accumulation may be related to TMA-associated polyserositis. ${ }^{6}$

\section{INCIDENCE AND PROGNOSIS}

In 2018, a retrospective study of 1,990 post-HSCT patients demonstrated a $13 \%$ incidence of definitive TMA and a $26 \%$ incidence of probable TA-TMA. ${ }^{8}$ Another study reported a $29 \%$ incidence of TA-TMA, with comparison of different diagnostic criteria among 672 patients. ${ }^{11}$

The median time to onset of post-HSCTTMA is 1.2 months and median survival time after TMA onset is 1.7 months. ${ }^{8}$ The 4 -year overall survival is reported as $55 \%$, nonrelapse mortality is $34 \%$, and relapse frequency is $10 \%$ in definitive TMA patients. ${ }^{8}$

The causes of death among TMA patients are difficult to differentiate due to overlapping conditions. The most common cause of death among patients who have responded to treatment is chronic graft-versus-host disease (cGVHD), followed by progression of malignancy and other reasons. ${ }^{12}$ The most common cause of death among patients who do not respond to TMA treatment is acute GVHD, followed by cGVHD, infection, hemorrhage, and other factors. ${ }^{12}$ Also, death related to veno-occlusive disease is significantly increased in definitive TMA patients. ${ }^{8}$

Proteinuria, elevated serum C5b-9 levels, unrelated donors, LDH-platelet ratio $>20$, schistocytes $>5$ to $10 /$ high-power field (hpf), and TMA without sirolimus are considered poor prognostic factors. ${ }^{13}$

\section{TREATMENT}

No proven and universally accepted treatment for TA-TMA exists currently. Several studies have recommended stopping or reducing CNIs and adding an additional GVHD prophylactic agent as first-line management. ${ }^{13}$ Mycophenolate mofetil, corticosteroids, and interleukin-2 (IL-2) inhibitors (daclizumab and basiliximab) are alternative treatments for GVHD prophylaxis. ${ }^{13}$ See Table 13.2 for current treatment options forTA-TMA.

\section{Eculizumab}

Eculizumab is a promising drug that blocks activation of the terminal complement pathway. Recent studies demonstrate that eculizumab reduces mortality and decreases long-term organ damage. ${ }^{17}$ Additionally, eculizumab normalizes the platelet count and improves $\mathrm{Cr}$ levels. ${ }^{17}$ Unfortunately, achievement of therapeutic dosages is challenging, and dosages must be tracked closely. 
Table 13.2 Current Treatment Options for Transplant-Associated Thrombotic Microangiopathy

\begin{tabular}{|l|l|l|l|}
\hline Treatment & Action Mechanism & Response & $\begin{array}{l}\text { Common Side } \\
\text { Effects }\end{array}$ \\
\hline Eculizumab & $\begin{array}{l}\text { Binds to C5 and blocks } \\
\text { complement cascade }\end{array}$ & $\begin{array}{l}67 \%^{14} \\
2014^{*}\end{array}$ & Infections $^{14}$ \\
\hline Defibrotide & $\begin{array}{l}\text { Polydisperse } \\
\text { oligonucleotide, protects } \\
\text { endothelium from damage }\end{array}$ & $\begin{array}{l}77 \%^{15} \\
2017\end{array}$ & $\begin{array}{l}\text { Hypotension, } \\
\text { diarrhea }^{13}\end{array}$ \\
\hline Rituximab & $\begin{array}{l}\text { Binds to CD20 and reduces } \\
\text { antibody production }\end{array}$ & $\begin{array}{l}67 \%^{16} \\
2015\end{array}$ & $\begin{array}{l}\text { Infusion } \\
\text { reactions, }_{\text {infections }}{ }^{14}\end{array}$ \\
\hline $\begin{array}{l}\text { Plasma } \\
\text { exchange }\end{array}$ & $\begin{array}{l}\text { Removes antibodies and } \\
\text { may also remove Ang-2 }\end{array}$ & $\begin{array}{l}\text { Bleeding, } \\
\text { infections }\end{array}$ \\
\hline $\begin{array}{l}\text { Note: Calcineurin inhibitors were reduced or stopped in all cases. } \\
\text { *Pediatric population }\end{array}$ & \\
\hline
\end{tabular}

Complement system activation can be confirmed with increased plasma C5b-9 levels (normal limit 119-175 $\mathrm{ng} / \mathrm{mL}$ ) and proteinuria (>30 mg/dL) before starting eculizumab treatment. ${ }^{18}$ Additionally, complement blockage with eculizumab is a risk factor for meningococcal infections and requires vaccination 2 weeks before starting treatment. However, the immunosuppressed state of patients may alter the effectiveness of the vaccine. Findings from a cohort study led to the recommendation of using antimicrobial prophylaxis for up to 8 weeks following the last dose of eculizumab, in order to prevent meningococcal infection, as the short period of exposure to eculizumab as well as the patients' immunosuppressed state precludes the necessity of a vaccine..$^{19}$

\section{Defibrotide}

Defibrotide, a polydeoxyribonucleotide, decreases endothelial destruction. Two multicenter retrospective studies encompassing 551 HSCT patients have reported a $55 \%$ response rate to defibrotide in those with grade 3-4TA-TMA. ${ }^{6}$

\section{Rituximab}

Rituximab is an anti-CD20 antibody that depletes antibodies and regulates the immune system. Case reports and small studies have demonstrated a clinical response to rituximab as monotherapy or in combination with defibrotide and therapeutic plasma exchange (TPE). However, current approaches have shifted toward utilizing complement-blocking therapies. 


\section{Therapeutic Plasma Exchange}

The 2005 TA-TMA consensus guidelines summarized 11 studies and reported a high mortality and poor response of patients to plasma exchange. ${ }^{20}$ However, early initiation (within 2-3 weeks after diagnosis) ofTPE can be beneficial for successful clearance of antibodies and cytokines. ${ }^{13} \mathrm{~A}$ recent study proposed that TPE can be used with rituximab in the presence of Factor $\mathrm{H}$ antibody, if a contraindication prevents the use of newer, more specifically targeted drugs. ${ }^{13}$

Other potential therapeutics, including newer complement system blocking agents, are being studied in phase 2 clinical trials. ${ }^{13}$

\section{REFERENCES}

1. Jodele S. Complement in pathophysiology and treatment of transplant-associated thrombotic microangiopathies. Semin Hematol. 2018;55(3):159-166. doi:10.1053/j.seminhematol.2018.04.003

2. Obut F, Kasinath V, Abdi R. Post-bone marrow transplant thrombotic microangiopathy. Bone Marrow Transpl. 2016;51(7):891-897. doi:10 .1038/bmt.2016.61

3. Wirtschafter E, VanBeek C, Linhares Y. Bone marrow transplant-associated thrombotic microangiopathy without peripheral blood schistocytes: a case report and review of the literature. Exp Hematol Oncol. 2018;7:14. doi:10.1186/s40164-018-0106-9

4. BrocklebankV, Wood KM, Kavanagh D. Thrombotic microangiopathy and the kidney. Clinical J Am Soc Nephrol. 2018;13(2):300-317. doi:10.2215/ CJN.00620117

5. Noris M, Galbusera M, Gastoldi S, et al. Dynamics of complement activation in aHUS and how to monitor eculizumab therapy. Blood. 2014;124(11):1715-1726. doi:10.1182/blood-2014-02-558296

6. Jodele $\mathrm{S}$, Laskin $\mathrm{BL}$, Dandoy $\mathrm{CE}$, et al. A new paradigm: diagnosis and management of HSCT-associated thrombotic microangiopathy as multi-system endothelial injury. Blood Rev. 2015;29(3):191-204. doi:10.1016/j.blre.2014.11.001

7. Qian $\mathrm{Q}$, Nath KA, WuY, et al. Hemolysis and acute kidney failure. Am J Kidney Dis. 2010;56(4):780-784. doi:10.1053/j.ajkd.2010.03.025

8. Postalcioglu M, Kim HT, Obut F, et al. Impact of thrombotic microangiopathy on renal outcomes and survival after hematopoietic stem cell transplantation. Biol Blood Marrow Transplant. 2018;24(11):2344-2353. doi:10.1016/j.bbmt.2018.05.010

9. Dowling MR, Li S, Dey BR, et al. Neurologic complications after allogeneic hematopoietic stem cell transplantation: risk factors and impact. Bone Marrow Transplant. 2018;53(2):199-206. doi:10.1038/ bmt.2017.239

10. Hobson EV, Craven I, Blank SC. Posterior reversible encephalopathy syndrome: a truly treatable neurologic illness. Perit Dial Int. 2012;32(6):590-594. doi:10.3747/pdi.2012.00152 
11. Cho B-S, Yahng S-A, Lee S-E, et al. Validation of recently proposed consensus criteria for thrombotic microangiopathy after allogeneic hematopoietic stem-cell transplantation. Transplantation. 2010;90(8):918-926. doi:10.1097/TP.0b013e3181f24e8d

12. Oran B, Donato $M$, Aleman A, et al. Transplant-associated microangiopathy in patients receiving tacrolimus following allogeneic stem cell transplantation: risk factors and response to treatment. Biol Blood Marrow Transplant. 2007;13(4):469-477. doi:10.1016/j.bbmt.2006.11.020

13. Khosla J, Yeh AC, Spitzer TR, Dey BR. Hematopoietic stem cell transplant-associated thrombotic microangiopathy: current paradigm and novel therapies. Bone Marrow Transplant. 2018;53(2):129-137. doi:10.1038/bmt.2017.207

14. Wanchoo R, Bayer RL, Bassil C, Jhaveri KD. Emerging concepts in hematopoietic stem cell transplantation-associated renal thrombotic microangiopathy and prospects for new treatments. Am J Kidney Dis. 2018;72(6):857-865. doi:10.1053/j.ajkd.2018.06.013

15. Yeates L, Slatter MA, Bonanomi S, et al. Use of defibrotide to treat transplant-associated thrombotic microangiopathy: a retrospective study of the Paediatric Diseases and Inborn Errors Working Parties of the European Society of Blood and Marrow Transplantation. Bone Marrow Transplant. 2017;52(5):762-764. doi:10.1038/bmt.2016.351

16. Kim SS, Patel M, Yum K, Keyzner A. Hematopoietic stem cell transplant-associated thrombotic microangiopathy: review of pharmacologic treatment options. Transfusion. 2015;55(2):452-458. doi:10.1111/ trf.12859

17. Vasu S, Wu H, Satoskar A, et al. Eculizumab therapy in adults with allogeneic hematopoietic cell transplant-associated thrombotic microangiopathy. Bone Marrow Transplant. 2016;51(9):1241-1244. doi:10.1038/ bmt.2016.87

18. Jodele S, Dandoy CE, Myers KC, et al. New approaches in the diagnosis, pathophysiology, and treatment of pediatric hematopoietic stem cell transplantation-associated thrombotic microangiopathy. Transfus Apher Sci. 2016;54(2):181-190. doi:10.1016/j.transci.2016.04.007

19. Jodele S, Dandoy CE, Danziger-Isakov L, et al. Terminal complement blockade after hematopoietic stem cell transplantation is safe without meningococcal vaccination. Biol Blood Marrow Transplant. 2016;22(7):1337-1340. doi:10.1016/j.bbmt.2016.03.032

20. Ho VT, Cutler C, Carter S, et al. Blood and marrow transplant clinical trials network toxicity committee consensus summary: thrombotic microangiopathy after hematopoietic stem cell transplantation. Biol Blood Marrow Transplant. 2005;11(8):571-575. doi:10.1016/j.bbmt.2005.06.001 